jow (which I have now pleasure in doing), and in stating briefly the course of the affection.

This seemingly hopeless case, which has, by what is certainly not a recognised method of treatment, given a most gratifying result, suggests the following conclusions. 1. There are probably some cases of atrophy of the optic nerve in which the hypodermic injection of pilocarpine would prove a valuable addition to the recognised methods of treatment. 2. These injections may, in suitable cases, be continued daily; for a long time, without lowering the ppatient's health. 3. Strychnine and pilocarpine may be administered subcutaneously at tho same time.

\section{CASES ILLUSTRATING THE VALUE OF THE BACILLUS-SEARCH.}

BY ARTHUR RANSOME, M.D., MANCHESTER.

GaSE I.-A boy, aged 8, seen with Dr. Mules, had suffered for several weeks from high temperature $\left(101^{\circ}\right.$ to $\left.103^{\circ}\right)$ with wasting and shortness of breath. The upper lobe of the left lung was consolidated, and gave moist sounds on auscultation. The expectoration was abundant, mucopurulent. Both bases were clear. No bacilli were discovered in the sputum after careful search; and, although negative eyidence on this point is of much less value than positive evidence, it was considered probable that the case was one of apicial pneumonia, and not phthisis. This opinion was justified several weeks later by complete recovery.

CASE Ir. - Also seen with Dr. Mules, was a man, aged 30, who had suffered from pleurisy, with effusion on the right side; for which aspiration had to be employed. He remained in bad health for several weeks afterwards, and his temperature continued high $\left(100^{\circ}\right.$ to $\left.102^{\circ}\right)$. There was dulness at the right base, with motst crepitation, and the expectoration was mucopurulent and abundant. There was also slight dulness at both apices. It was difficult to say how far his condition was to be explained by the previous plourisy until the sputum was examined, and found to contain abundant bacilli. He subsequently became insane, and died, a few months afterwards, of phthisis.

CASE III. - A woman attending the Hospital for Consumption, Mamchester, suffering from constant cough, abundant :expectoration, and night-sweats, was rapidly emaciating. Although no physical gigns of phthisis were discoverable, the sputum was examined; no bacilli were found, and a favourable prognosis was given. She afterwards recovered, after several month' illness.

This case may serve as an example of several others of a similar gharacter.

CASE IV.-A gentleman, residing at Alderley, had suffered from an occasional hæmoptysis for two years. He was rather thin, and had ognstant slight cough, but had not lost weight of late. There was slight dulness at the right apex, and harsh breathing; no moist sounds. He had been pronounced free from tubercular disease by two eminent London physicians. An examination of some blood-stained spatum on his handkerchief gave several bacilli, and he was advised to winter in the Riviera.

CAsE V.-A married lady, aged 30, had suffered for three years from symptoms of phthisis, and it had become a question whether she might prudently winter at Davos. She had occasional hæmoptysis, and there were vomicæ at the bases of both lungs ; but her weight, nnder the use of iodoform, had increased during the last few months, and her expectoration had diminished. Examination of the sputum showed comparatively few bacilli. It was, therefore, considered pro. bable that the disease had assumed a chronic form; and that she might go to the Engadine in September. When there, she had a sharp attack of rheumatic fever; but, in spite of this, $I$ learn that she has gained weight, and that the lung-disease seems to have made some improvement.

CASE VI.-A youth, aged 17, had pneumonia at the base of the left lung, and afterwards pleurisy, . ending in empyema. His familyhistory was unfavourable; but no physical signs of tubercle could be getected, and he had very little cough and expectoration. A small portion of rusty pneumonic sputum was secured, and a few bacilli. pre found. The empyema was treated by free incision, drainage, and antiseptic irrigation, etc., and the cavity is closing up; but he romains still in a precarious condition.

CASE VII. - A girl, aged 15, was seized three months ago with onteric fever, the result of impure milk, After a lapse of three weeks, she had a second rigor, which ushered in a second attack of fever, with dianchoea and rose-spots at the due interval. In rather more than three weeks from the commencement of her illness, the fever subsided for a week, and then began a third time. A third crop of rosespoto appeared a fow days afterwards, and continued to show themselves for ten days. She had suffered from cough throughout the illness; and there was congestion of the left base. She was greatly emaciated; and, although the third outbreak of the rash made it pretty certain that we had to do with the extraordinary occurrence of a second relapse into fever, it was thought desirable to discover whether tubercular disease was also present. The sputtum was; therefore, carefully examined; and, no bacilli being found, our anxiety on this score was relieved. The case is now convalescent.

OAst vir.-A farmer, aged 40, had pulmonary pneumonia, with effuision on the left side. After a month or two; the fluid diminished without aspiration, and he improved in general health $\xi$ but there remained some dulness at the base of the lung. Four mbnthe after his first seizure, he-began to lose weight, and was feverish, the evening temperature being $103^{\circ}$; and had aphthæ in the throat and mouth. Being badly hotised and badly nursed, he was persuaded to come into the Altrincham Hospital. An exploratory aspiration was made, in search of any possiblelimited collection of pus, but without success. His sputum was then examined; and, ialthough no bacilli were found, there was an abundance of a minute micrococcus, and it became evident that we had to do with destructive pneumonic suppuration. He died shortly. afterwards.

\section{THERAPEUTIC MEMORANDA.}

\section{THE TREATMENT OF TUBERCULOSIS.}

Dr. G. Hunter Mackenzie, in the Journal of October 11th, page 711 , comments on the absence of any records of the effect of treatiment upon the bacilli of tubercle; and he remarks that; in the reports of my paper read at the Copenhagen meeting of the International Congress, no reference is made to the effect of iodoform upon the bacilli in the sputum. When the paper was called for, the time of the meeting was so limited that only a small portion was read, and only a small fraction of this was reported. Accordingly, Dr. Mackenzie is not yet in a position to state what evidence is contained in the paper and he will, no doubt, read with interest the details therein contrined, when the paper has been published in the Transactions of the Congress. In the meantime, I may be allowed to state that the action of iodoform on the bacilli in the sputum was a point to which I had given special attention; and many of my cases appear to show that the bacilli diminish in number, and disappear from the sputum, under the influence of this drug. In several of the cases, all active symptoms had subsided, and expectoration ceased, so that no sputum could be obtained for examination. The following case is the most striking and satisfactory one in its results.

The patient was a female, aged 39. Her husband died of eonsumption two years ago; a son was now ill with tubercular phthisis (sputum containing bacilli). She formerly weighed $8 \mathrm{st}$. $13 \mathrm{lb}$.; her present weight was 6 st. $13 \mathrm{lb}$. She had had cough for five months; the sputum contained numerous typical bacilli. She had hectic temperature; highest, $101^{\circ}$. There was dulness and crepitation at the left apex. She commenced iodoform on September 3ra, 1883, one grain every four hours, increased gradually up to five grains on September 22nd. Steady improvement ensued, and on October 16th she had gained strength; no crepitation could be heard, there was little expectoration, and the temperature normal. On November 27 th, the weight was 8 stone, and her general health good. The ipeioform was then discontinued. On December 11th, her weight was 8 st $4 \mathrm{lb}$. No expectoration could be obtained, as she had long ceased to expectorate, and she considered herself quite well. There was no dulness at the apex of the lung, and no other evidence of disease.

Another case was as follows.

A male patient, aged 29 , had had cough for five years, and hæmoptysis and wasting for threo weeks; his weight was 11 st. 2 lbs. on March 28th, 1883. There were evidences of disease at the apex of the right lung. Temperature, $98^{\circ}$. His sputum contained numerous tabereulous bacilli. He commenced iodoform on April 10th, one grain every four hours. On April 23rd, there was little cough and slight expectoration, the latter containing anly a very few bacilli. The iodo. form was increased to two grains, and was cantinued till June 4 th. The weight had steadily increased ap to 12 st. $3 \mathrm{lbs}$. on June 26 th, when the patient appeared to be well, and had no cough or expectoration, although there was still some dulness, and coarse breath-sounds could be heard at the right apex.

$A$ third case was that of William M, aged 34, who, after fourteen weeks' cough, had lost two stone in weight; ho had pneumothorax at tho 